\title{
BL Lac object 1ES 0647+250, a decade of MWL observations
}

\section{J. Otero-Santos, ${ }^{a, *}$ D. Dorner, ${ }^{b}$ D. Morcuende, ${ }^{c}$ D. Paneque,${ }^{d}$ V. Fallah Ramazani, ${ }^{e}$ E. Prandini $^{f}$ and G. Bonnoli ${ }^{g}$ on behalf of the MAGIC ${ }^{\dagger}$ and Fermi-LAT Collaborations and MWL partners}

${ }^{a}$ Instituto de Astrofísica de Canarias (IAC) and Universidad de La Laguna (ULL), San Cristóbal de La Laguna, Spain

${ }^{b}$ Universität Würzburg, Institut für Theoretische Physik und Astrophysik, Würzburg, Germany

${ }^{c}$ IPARCOS Institute and EMFTEL Department, Universidad Complutense de Madrid, Madrid, Spain

${ }^{d}$ Max Planck Institute for Physics, München, Germany

${ }^{e}$ Finnish Centre for Astronomy with ESO (FINCA), University of Turku, Finland and Ruhr-Universität, Fakultät für Physik und Astronomie, Astronomisches Institut (AIRUB), Bochum, Germany

${ }^{f}$ University of Padova, Padova, Italy

${ }^{g}$ Instituto de Astrofísica de Andalucía (IAA), Granada, Spain

E-mail: joteros@iac.es

The high-peaked BL Lac object 1ES 0647+250 is one of the few distant blazars detected at veryhigh-energy (VHE, $E>100 \mathrm{GeV}$ ) $\gamma$ rays during non-flaring activity. Its redshift is still uncertain, with a recently proposed lower limit of $z>0.29$. This blazar was first detected by the MAGIC telescopes between 2009 and 2011 during its low state, displaying a flux of around $2 \%$ of the Crab Nebula above $100 \mathrm{GeV}$, but it has shown several periods of high activity, where the VHE $\gamma$-ray flux increased by more than one order of magnitude. The VHE spectra of four observed periods are characterized and the redshift of this source is estimated using the HE and VHE spectral shapes. The multi-wavelength data set collected from 2009 to 2020 introduced in this work will serve as the seed for further studies, including detailed studies of the broad-band spectral energy distribution for different activity levels and multi-band variability and correlation studies.

$37^{\text {th }}$ International Cosmic Ray Conference (ICRC 2021)

July 12th - 23rd, 2021

Online - Berlin, Germany

\section{*Presenter}

${ }^{\dagger}$ A complete list of the MAGIC Collaboration authors can be found at the end of the proceedings 


\section{Introduction}

Blazars are the most numerous sources in the extragalactic $\gamma$-ray sky. They are active galactic nuclei (AGNs) with a relativistically boosted jet pointing towards the Earth. Their emission extends from radio to very-high-energy (VHE, $\mathrm{E}>100 \mathrm{GeV}$ ) $\gamma$ rays. They show a characteristic doublebump shape in their spectral energy distribution (SED). The low-energy bump is dominated by non-thermal synchrotron emission of the relativistic electrons moving under the influence of a magnetic field through the jet. The high-energy bump is typically explained by a leptonic scenario, where low energy photons are scattered via inverse Compton (IC) by the same population of electrons. The low-energy photons can be injected from outside the jet (external Compton, EC [1]), or they can have their origin in the jet via synchrotron radiation (synchrotron self Compton, SSC [2]). However, this is still under debate and more complex emission models or hadronic scenarios are sometimes needed to explain the $\gamma$-ray emission from these sources.

Among blazars, BL Lac type objects represent most of the sources detected in the VHE band. They can be sub-classified into different types according to the frequency of the synchrotron peak [3]: Low-energy peaked BL Lacs (LBLs, $v_{\text {peak }}<10^{14} \mathrm{~Hz}$ ), intermediate-energy peaked BL Lacs (IBLs, $10^{14}<v_{\text {peak }}<10^{15} \mathrm{~Hz}$ ), and high-energy peaked BL Lacs (HBLs, $v_{\text {peak }}>10^{15} \mathrm{~Hz}$ ). In the last years, a new category of extreme-high-energy peaked BL Lacs (EHBLs, $v_{\text {peak }}>10^{17} \mathrm{~Hz}$ ) was suggested [4].

1ES $0647+250$ is a BL Lac type object classified as a HBL. Its redshift is still under debate. Several estimations of its distance were made in the past (e.g. $\mathrm{z}=0.41$ based on the detection of the underlying host galaxy in the near-infrared band, see [5]). However, the exact value is still unknown. The most recent measurement led to a lower limit of $\mathrm{z}>0.29$ based on the minimum equivalent width of absorption features expected from the host galaxy [6]. The source was first detected by MAGIC between 2009 and 2011 during a low flux state [7]. Thus, it is one of the few distant blazars detected in a non-flaring state. Later, it was observed on several occasions in an enhanced activity state. In addition, it was also regularly observed in radio, optical and X-ray bands, and at high-energy (HE) $\gamma$ rays by the Fermi-LAT telescope. Here, we introduce the results of the MAGIC and multi-wavelength (MWL) analysis of more than ten years of data from this source.

\section{MAGIC Observations}

MAGIC is a stereoscopic pair of 17-meter Imaging Atmospheric Cherenkov Telescopes (IACTs) located on the Canary Island of La Palma, at the Roque de los Muchachos observatory ( $2200 \mathrm{~m}$ a.s.1.). They operate in the VHE $\gamma$ ray band of the electromagnetic spectrum, between $\sim 50 \mathrm{GeV}$ up to a few TeVs, with a sensitivity above $0.1 \mathrm{TeV}$ of about $1.5 \%$ of the Crab Nebula flux after a 50-h long observation (see Table A.5 in [8]).

1ES $0647+250$ was observed by the MAGIC telescopes between November 2009 and December 2020 for a total of $\sim 45 \mathrm{~h}$, mainly under dark conditions and at a zenith angle below $35^{\circ}$. The observations performed between November 2009 and March 2011 correspond to its non-flaring state. These observations led to the detection of 1ES $0647+250$ between 2009 and 2011 with a significance of $5.5 \sigma$. Later, several observations of different high states of the source were carried out following up on enhanced activities reported at optical and X-ray wavelengths in the years 2014, 

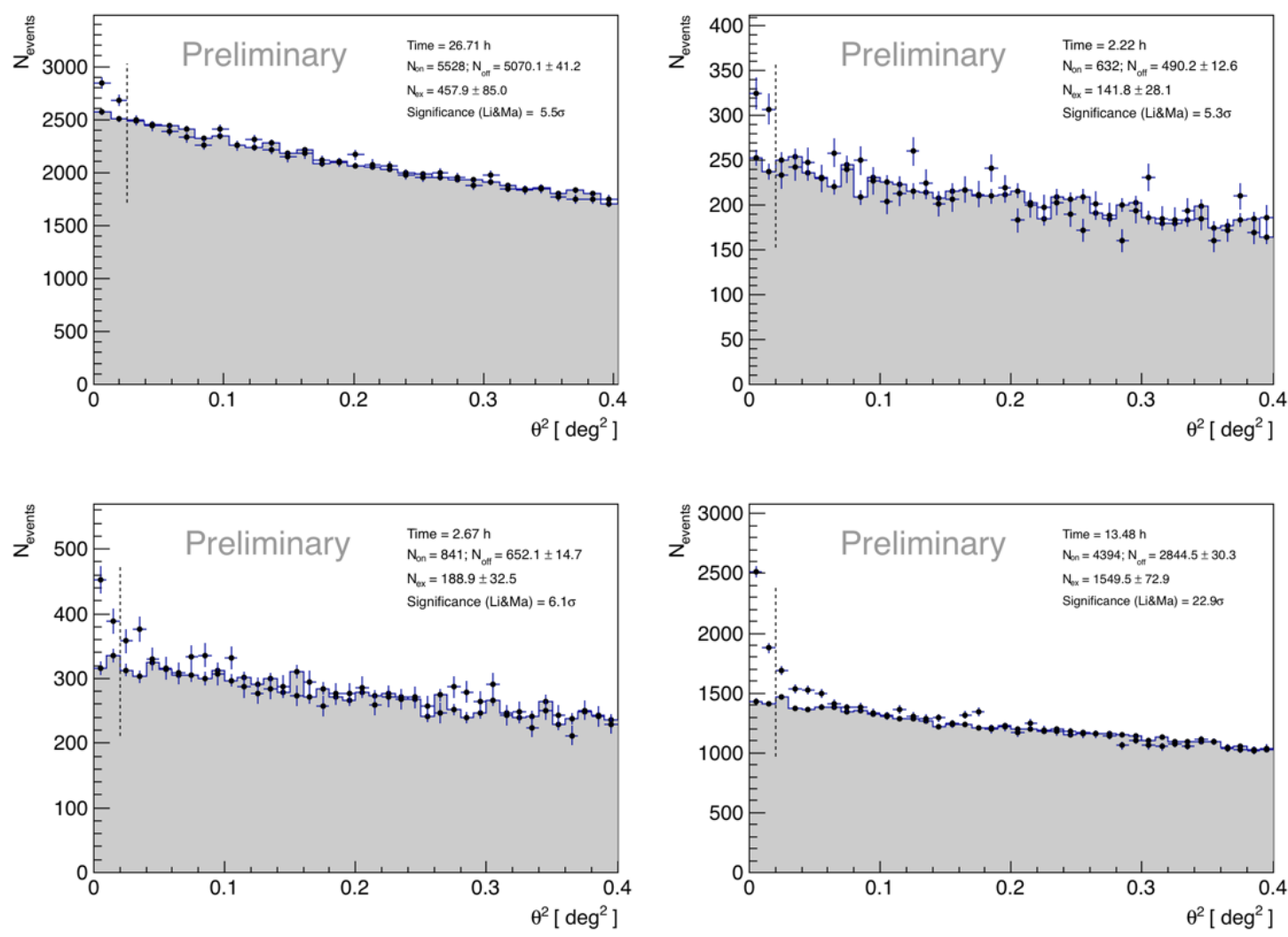

Figure 1: Theta-squared $\left(\theta^{2}\right)$ and normalized background distributions of 1ES $0647+250$ for the different analysis epochs. Top left: November 2009 to March 2011 (low state). Top right: November 2014 (high state). Bottom left: December 2019 (high state). Bottom right: December 2020 (high state). Signal events are represented by filled circles highlighted with crosses, and the background distributions are shown with the filled area.

2019 and 2020 [9-11]. The observations performed in 2014, 2019 and 2020 found the source in an elevated state in VHE, and yielded detections with significances of $5.3 \sigma, 6.1 \sigma$ and $22.9 \sigma$, respectively (see Fig. 1). In all cases, the data were analyzed using the MAGIC analysis and reconstruction software (MARS) [12], and the significance of the detection is calculated following Eq. 17 in [13].

\section{Multi-Wavelength Observations}

The VHE observations were complemented with the HE $\gamma$-ray analysis of the data provided by the Large Area Telescope (LAT) on board the Fermi satellite [14]. The Fermi-LAT data were analyzed using the standard Fermi analysis software tools (version v11r07p00), and the P8R3_SOURCE_V2 response functions. We used events from 0.3 to $300 \mathrm{GeV}$ selected within a $15^{\circ}$ region of interest (ROI) centered on $1 \mathrm{ES} 0647+250$, and having a zenith distance below $100^{\circ}$ to avoid contamination from the Earth's limb. The diffuse Galactic and isotropic components were 


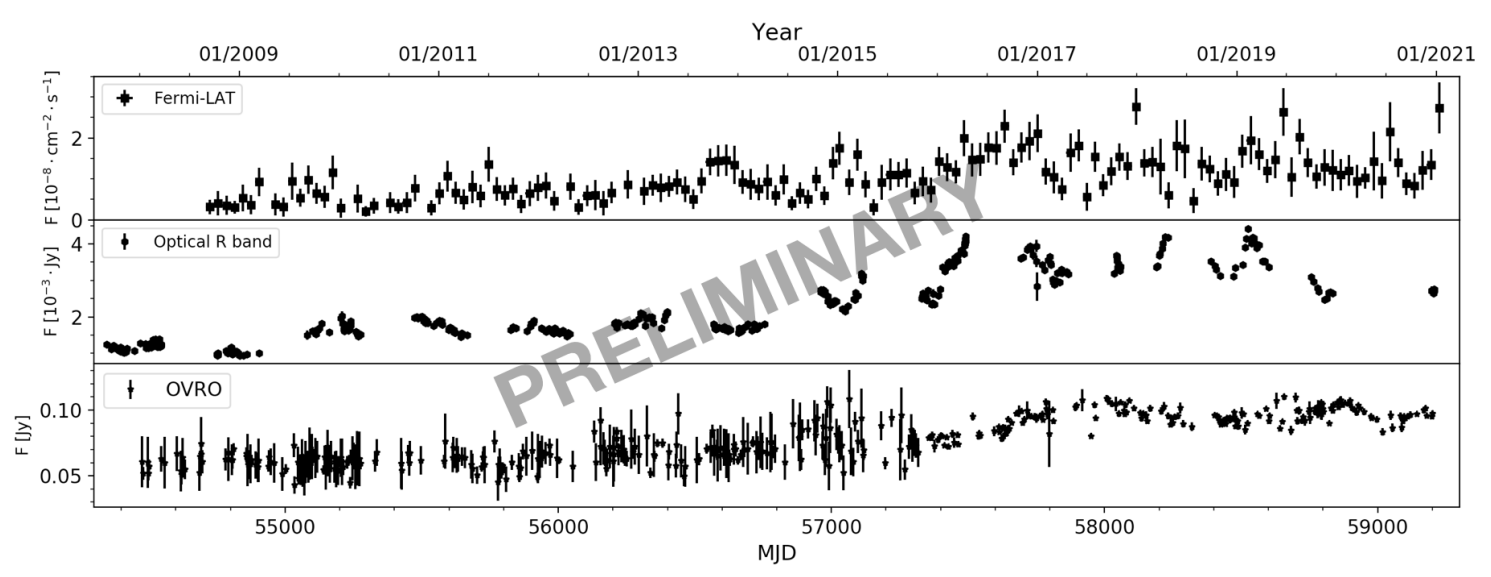

Figure 2: MWL light curves of 1ES 0647+250. From top to bottom: Fermi-LAT (0.3-300 GeV, monthly binned); optical R band; and OVRO (15 GHz). All the optical data were taken with the KVA telescope of the Tuorla blazar monitoring program except from the observations of the 2020 flare. The decrease in the error bars of the OVRO light curve after 2016 is due to a major upgrade of the telescope.

modelled with the files gll_iem_v06.fits and iso_P8R3_SOURCE_V2.txt. respectively ${ }^{1}$. The source was observed to be very weak in the HE regime. In order to have significant detections, the HE $\gamma$-ray light curve was generated using a 30-day binning.

We have collected simultaneous X-ray and ultraviolet (UV) data from the Neil Gehrels Swift satellite and its instruments, the X-Ray Telescope (XRT) [15] and the Ultra-Violet and Optical Telescope (UVOT, see [16]). 1ES 0647+250 was observed by Swift with both instruments for a total of 70 times during the 11-year interval: 38 observations between 2010 and 2012; 5 during the 2014 flare; 9 during December 2020; and 18 during 2020 (8 out of 18 during December 2020).

This blazar has also been the target of several MWL and monitoring programs. It was observed regularly by the Tuorla blazar monitoring program ${ }^{2}$ in the R-band from December 2002 to December 2019. This monitoring was performed with the $60 \mathrm{~cm}$ Kungliga Vetenskapsakademien (KVA) telescope, located in La Palma. Later in 2020, the last high state was followed by the telescopes of Las Cumbres (LCOGT) [17], the PIRATE telescope and the Liverpool telescope. Finally, radio data from the Owens Valley Radio Observatory (OVRO) blazar monitoring program ${ }^{3}$ were also included to cover radio wavelengths [18]. The HE $\gamma$-ray, R-band optical and radio light curves of 1ES 0647+250 used in this work are reported in Fig. 2.

\section{Results}

We present the characterization of the VHE gamma-ray emission of 1ES 0647+250 during the different epochs, that correspond to different observed states. We estimated and compared the VHE spectrum and SED of each period. The SEDs of the low state and the flaring states from 2014 and 2019 were modelled with a power-law function $\left(d N / d E=f_{0} \cdot\left(E / E_{0}\right)^{\alpha}\right)$, while for the spectrum of 2020 , a $3 \sigma$ preference for a log-parabola function $\left(d N / d E=f_{0} \cdot\left(E / E_{0}\right)^{\alpha+\beta \cdot \log \left(E / E_{0}\right)}\right)$

\footnotetext{
${ }^{1}$ https://fermi.gsfc.nasa.gov/ssc/data/access/lat/BackgroundModels.html

${ }^{2}$ http://users.utu.fi/kani/1m/

${ }^{3}$ https://www. astro.caltech.edu/ovroblazars/
} 


\begin{tabular}{cccc}
\hline \multirow{2}{*}{ Period } & $F(E>100 \mathrm{GeV})$ & \multicolumn{2}{c}{ Spectral parameters } \\
\cline { 3 - 4 } & {$[\%$ Crab Flux $]$} & $E_{0}[\mathrm{GeV}]$ & Spectral index \\
\hline $2009-2011$ & $2.0 \pm 0.5$ & 190 & $\alpha=-3.1 \pm 0.4$ \\
\hline 2014 & $3.4 \pm 1.6$ & 100 & $\alpha=-3.3 \pm 0.7$ \\
\hline 2019 & $8.0 \pm 1.8$ & 100 & $\alpha=-3.7 \pm 0.6$ \\
\hline 2020 & $15.0 \pm 1.0$ & 100 & $\begin{array}{c}\alpha=-3.2 \pm 0.2 \\
\beta=-1.9 \pm 0.7\end{array}$ \\
\hline
\end{tabular}

Table 1: Integrated fluxes above $100 \mathrm{GeV}$ in Crab Units and spectral parameters for each observed period.

was observed. We also estimated the VHE gamma-ray integral flux above $100 \mathrm{GeV}$ considering the spectral shapes already commented. The source was first detected during its non-flaring state between 2009 and 2011. The integral flux above $100 \mathrm{GeV}$ during this period was estimated to be $(9.7 \pm 2.4) \cdot 10^{-12} \mathrm{~cm}^{-2} \mathrm{~s}^{-1}$. The first enhanced flux state, detected in November 2014, showed a flux increase of a factor $\sim 1.7$, with an average flux of $(1.6 \pm 0.8) \cdot 10^{-11} \mathrm{~cm}^{-2} \mathrm{~s}^{-1}$. Later in 2019, the source displayed a brightening of almost a factor 4 w.r.t. the low state, and an average emission of $(3.8 \pm 0.9) \cdot 10^{-11} \mathrm{~cm}^{-2} \mathrm{~s}^{-1}$. Finally, 2020 corresponds to the brightest state of this blazar at VHE. During this enhanced state, the source increased its emission by a factor $\sim 7.5$ w.r.t. its non-flaring state. The average flux during this flare was estimated to be $(7.1 \pm 0.5) \cdot 10^{-11} \mathrm{~cm}^{-2} \mathrm{~s}^{-1}$. The flux values and spectral parameters of the individual flux states are reported in Table 1.

The observed spectrum is affected by the interaction with the extragalactic background light (EBL) depending on the energy of the gamma rays detected and the distance to the source. Based on the HE and VHE spectral shapes, we performed an estimation of the redshift for this blazar following the method proposed by [19], taking advantage of the fact that the observed spectral shape changes with the distance to the object. First, iterating over fine steps of redshift, a limit value, $z *$, is calculated under the assumption that the intrinsic VHE spectrum cannot be harder than the HE spectrum. With this premise, $z *$ is determined as the redshift at which both spectral slopes are equal. For sources with well-known distances, an empirical linear relation between $z *$ and the true redshift is derived. Therefore, the reconstructed redshift $z_{\text {rec }}$ is obtained by using this relation and the $z *$ previously estimated. We applied this method to the spectrum of 1ES 0647+250 from 2020 (see Table 1) due to its smaller errors compared to the other periods. The Fermi-LAT spectral index derived using simultaneous data to the MAGIC observations from 2020 is $-1.58 \pm 0.17$.

Alternatively, following the method presented in [20], a 95\% confidence level (CL) upper limit (UL) to the redshift was also obtained through the joint fit of the Fermi-LAT and MAGIC spectra iterating over small redshift steps. The absorption by the EBL model described in [21] was taken into account. The spectrum in the GeV-TeV energy range was forced to be concave assuming that the MAGIC spectrum is modelled with a log-parabola function. Considering a $15 \%$ systematic uncertainty in the absolute energy and flux normalization scale based on the study performed in [8], the $95 \%$ CL UL to the redshift is 0.81 .

The results from the redshift estimation described above are shown in Table 2. The reconstructed redshift is compatible with previous measurements (e.g. $z=0.41$ by [5] based on the host galaxy measurements). 


\begin{tabular}{ccc}
\hline$z_{\text {rec }}$ & $z *$ & $95 \%$ CL UL \\
\hline $0.45 \pm 0.05$ & $0.75 \pm 0.11$ & 0.81 \\
\hline
\end{tabular}

Table 2: Estimated redshift and redshift upper limit of 1ES 0647+250 based on HE and VHE data.

\section{Conclusions}

We have introduced the MAGIC and MWL analysis of the BL Lac type object 1ES 0647+250. We reported the detection of this distant source during a non-flaring activity, and after, during three different enhanced states with increasing flux. We have characterized the VHE spectrum of the source in both its low and high activity levels. An estimation of the redshift was performed, and the reconstructed distance was found to be in agreement with previous measurements. Moreover, 11 years of MWL data were collected in order to study the long-term evolution of this blazar.

More details on the results of the 11-year data set MAGIC and MWL analysis of 1ES 0647+250 will be published soon in a dedicated paper by the MAGIC Collaboration [22].

\section{Acknowledgments}

MAGIC: We acknowledge the support from the agencies and organizations listed here: https:// magic.mpp.mpg.de/acknowledgments_ICRC2021/.

Fermi-LAT: The Fermi-LAT Collaboration acknowledges generous ongoing support from a number of agencies and institutes that have supported both the development and the operation of the LAT as well as scientific data analysis. These include the National Aeronautics and Space Administration and the Department of Energy in the United States, the Commissariat à l'Energie Atomique and the Centre National de la Recherche Scientifique / Institut National de Physique Nucléaire et de Physique des Particules in France, the Agenzia Spaziale Italiana and the Istituto Nazionale di Fisica Nucleare in Italy, the Ministry of Education, Culture, Sports, Science and Technology (MEXT), High Energy Accelerator Research Organization (KEK) and Japan Aerospace Exploration Agency (JAXA) in Japan, and the K. A. Wallenberg Foundation, the Swedish Research Council and the Swedish National Space Board in Sweden.

\section{References}

[1] C.D. Dermer and R. Schlickeiser, On the Location of the Acceleration and Emission Sites in Gamma-Ray Blazars, Astrophysical Journal Supplement 90 (1994) 945.

[2] G. Ghisellini, F. Tavecchio, L. Foschini, G. Ghirland a, L. Maraschi and A. Celotti, General physical properties of bright Fermi blazars, Monthly Notices of the Royal Astronomical Society 402 (2010) 497 [0909.0932].

[3] A.A. Abdo, M. Ackermann, M. Ajello, W.B. Atwood, M. Axelsson, L. Baldini et al., Spectral Properties of Bright Fermi-Detected Blazars in the Gamma-Ray Band, The Astrophysical Journal 710 (2010) 1271 [1001.4097].

[4] L. Costamante, G. Ghisellini, P. Giommi, G. Tagliaferri, A. Celotti, M. Chiaberge et al., Extreme synchrotron BL Lac objects. Stretching the blazar sequence, Astronomy and Astrophysics 371 (2001) 512 [astro-ph/0103343]. 
[5] J.K. Kotilainen, T. Hyvönen, R. Falomo, A. Treves and M. Uslenghi, The host galaxy of the BL Lacertae object 1ES 0647+250 and its imaging redshift, Astronomy and Astrophysics 534 (2011) L2 [1109.0627].

[6] S. Paiano, M. Landoni, R. Falomo, A. Treves, R. Scarpa and C. Righi, On the Redshift of TeV BL Lac Objects, The Astrophysical Journal 837 (2017) 144 [1701.04305].

[7] B. De Lotto and MAGIC Collaboration, The MAGIC telescopes: performance, results and future perspectives, in Journal of Physics Conference Series, vol. 375 of Journal of Physics Conference Series, p. 052021, July, 2012, DOI.

[8] J. Aleksić, S. Ansoldi, L.A. Antonelli, P. Antoranz, A. Babic, P. Bangale et al., The major upgrade of the MAGIC telescopes, Part II: A performance study using observations of the Crab Nebula, Astroparticle Physics 72 (2016) 76 [1409. 5594].

[9] S. Kiehlmann, E. Lindfors and V. Larionov, Optical flare of the TeV blazar 1ES 0647+250, The Astronomer's Telegram 6726 (2014) 1.

[10] B. Kapanadze, The Higherst Historical 0.3-10 keV State of the TeV Source 1ES 0647+250, The Astronomer's Telegram 13324 (2019) 1.

[11] B. Kapanadze, Another Strong X-Ray flare in the TeV-Detected Blazar 1ES 0647+250, The Astronomer's Telegram 14264 (2020) 1.

[12] R. Zanin, E. Carmona, J. Sitarek, P. Colin, K. Frantzen, M. Gaug et al., MARS, The MAGIC Analysis and Reconstruction Software, in International Cosmic Ray Conference, vol. 33 of International Cosmic Ray Conference, p. 2937, Jan., 2013.

[13] T.P. Li and Y.Q. Ma, Analysis methods for results in gamma-ray astronomy., The Astrophysical Journal 272 (1983) 317.

[14] W.B. Atwood, A.A. Abdo, M. Ackermann, W. Althouse, B. Anderson, M. Axelsson et al., The Large Area Telescope on the Fermi Gamma-Ray Space Telescope Mission, The Astrophysical Journal 697 (2009) 1071 [0902.1089].

[15] D.N. Burrows, J.E. Hill, J.A. Nousek, A.A. Wells, G. Chincarini, A.F. Abbey et al., The Swift X-Ray Telescope, in X-Ray and Gamma-Ray Instrumentation for Astronomy XIII, K.A. Flanagan and O.H.W. Siegmund, eds., vol. 5165 of Society of Photo-Optical Instrumentation Engineers (SPIE) Conference Series, pp. 201-216, Feb., 2004, DOI.

[16] P.W.A. Roming, T.E. Kennedy, K.O. Mason, J.A. Nousek, L. Ahr, R.E. Bingham et al., The Swift Ultra-Violet/Optical Telescope, Space Science Reviews 120 (2005) 95 [astro-ph/0507413].

[17] T.M. Brown, N. Baliber, F.B. Bianco, M. Bowman, B. Burleson, P. Conway et al., Las Cumbres Observatory Global Telescope Network, Publications of the Astronomical Society of the Pacific 125 (2013) 1031 [1305.2437].

[18] J.L. Richards, W. Max-Moerbeck, V. Pavlidou, O.G. King, T.J. Pearson, A.C.S. Readhead et al., Blazars in the Fermi Era: The OVRO $40 \mathrm{~m}$ Telescope Monitoring Program, The Astrophysical Journal Supplement Series 194 (2011) 29 [1011.3111].

[19] E. Prandini, G. Bonnoli, L. Maraschi, M. Mariotti and F. Tavecchio, Constraining blazar distances with combined Fermi and TeV data: an empirical approach, Monthly Notices of the Royal Astronomical Society 405 (2010) L76 [1003 . 1674]. 
[20] W.A. Rolke, A.M. López and J. Conrad, Limits and confidence intervals in the presence of nuisance parameters, Nuclear Instruments and Methods in Physics Research Section A: Accelerators, Spectrometers, Detectors and Associated Equipment 551 (2005) 493.

[21] A. Domínguez, J.R. Primack, D.J. Rosario, F. Prada, R.C. Gilmore, S.M. Faber et al., Extragalactic background light inferred from AEGIS galaxy-SED-type fractions, Monthly Notices of the Royal Astronomical Society 410 (2011) 2556 [1007 . 1459].

[22] MAGIC Collaboration, J. Otero-Santos, D. Dorner, D. Morcuende, D. Paneque, V. Fallah Ramazani et al., Long term multi-wavelength study of 1ES 0647+250, Astronomy and Astrophysics, in preparation (2021). 


\section{Full Authors List: MAGIC Collaboration}

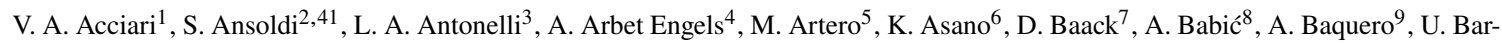
res de Almeida ${ }^{10}$, J. A. Barrio ${ }^{9}$, I. Batković ${ }^{11}$, J. Becerra González ${ }^{1}$, W. Bednarek ${ }^{12}$, L. Bellizzi ${ }^{13}$, E. Bernardini ${ }^{14}$, M. Bernardos ${ }^{11}$, A. Berti ${ }^{15}$, J. Besenrieder ${ }^{15}$, W. Bhattacharyya ${ }^{14}$, C. Bigongiari ${ }^{3}$, A. Biland ${ }^{4}$, O. Blanch ${ }^{5}$, H. Bökenkamp ${ }^{7}$, G. Bonnoli ${ }^{16}$, Ž. Bošnjak ${ }^{8}$, G. Busetto ${ }^{11}$, R. Carosi $^{17}$, G. Ceribella ${ }^{15}$, M. Cerruti ${ }^{18}$, Y. Chai ${ }^{15}$, A. Chilingarian ${ }^{19}$, S. Cikota ${ }^{8}$, S. M. Colak ${ }^{5}$, E. Colombo ${ }^{1}$, J. L. Contreras ${ }^{9}$, J. Cortina ${ }^{20}$, S. Covino ${ }^{3}$, G. D’Amico ${ }^{15,42}$, V. D’Elia ${ }^{3}$, P. Da Vela ${ }^{17,43}$, F. Dazzi ${ }^{3}$, A. De Angelis ${ }^{11}$, B. De Lotto ${ }^{2}$, M. Delfino ${ }^{5,44}$, J. Delgado ${ }^{5,44}$, C. Delgado $M_{e n d e z}^{20}$, D. Depaoli ${ }^{21}$, F. Di Pierro ${ }^{21}$, L. Di Venere ${ }^{22}$, E. Do Souto Espiñeira ${ }^{5}$, D. Dominis Prester $^{23}$, A. Donini ${ }^{2}$, D. Dorner ${ }^{24}$, M. Doro ${ }^{11}$, D. Elsaesser ${ }^{7}$, V. Fallah Ramazani ${ }^{25,45}$, A. Fattorini ${ }^{7}$, M. V. Fonseca ${ }^{9}$, L. Font ${ }^{26}$, C. Fruck ${ }^{15}$, S. Fukami ${ }^{6}$, Y. Fukazawa ${ }^{27}$, R. J. García López ${ }^{1}$, M. Garczarczyk ${ }^{14}$, S. Gasparyan ${ }^{28}$, M. Gaug ${ }^{26}$, N. Giglietto ${ }^{22}$, F. Giordano ${ }^{22}$, P. Gliwny ${ }^{12}$, N. Godinović ${ }^{29}$, J. G. Green ${ }^{3}$, D. Green ${ }^{15}$, D. Hadasch ${ }^{6}$, A. Hahn ${ }^{15}$, L. Heckmann ${ }^{15}$, J. Herrera ${ }^{1}$, J. Hoang ${ }^{9,46}$, D. Hrupec ${ }^{30}$, M. Hütten ${ }^{15}$, T. Inada ${ }^{6}$, K. Ishio ${ }^{12}$, Y. Iwamura ${ }^{6}$, I. Jiménez Martínez ${ }^{20}$, J. Jormanainen ${ }^{25}$, L. Jouvin ${ }^{5}$, M. Karjalainen ${ }^{1}$, D. Kerszberg ${ }^{5}$, Y. Kobayashi' ${ }^{6}$, H. Kubo ${ }^{31}$, J. Kushida ${ }^{32}$, A. Lamastra ${ }^{3}$, D. Lelas ${ }^{29}$, F. Leone ${ }^{3}$, E. Lindfors ${ }^{25}$, L. Linhoff ${ }^{7}$, S. Lombardi ${ }^{3}$, F. Longo ${ }^{2,47}$, R. López-Coto ${ }^{11}$, M. López-Moya ${ }^{9}$, A. López-Oramas ${ }^{1}$, S. Loporchio ${ }^{22}$, B. Machado de Oliveira Fraga $^{10}$, C. Maggio ${ }^{26}$, P. Majumdar ${ }^{33}$, M. Makariev ${ }^{34}$, M. Mallamaci ${ }^{11}$, G. Maneva ${ }^{34}$, M. Manganaro ${ }^{23}$, K. Mannheim ${ }^{24}$, L. Maraschi ${ }^{3}$, M. Mariotti ${ }^{11}$, M. Martínez ${ }^{5}$, D. Mazin ${ }^{6,15}$, S. Menchiari ${ }^{13}$, S. Mender ${ }^{7}$, S. Mićanović2 ${ }^{23}$, D. Miceli ${ }^{2,49}$, T. Miener ${ }^{9}$, J. M. Miranda ${ }^{13}$, R. Mirzoyan ${ }^{15}$, E. Molina ${ }^{18}$, A. Moralejo ${ }^{5}$, D. Morcuende ${ }^{9}$, V. Moreno ${ }^{26}$, E. Moretti ${ }^{5}$, T. Nakamori ${ }^{35}$, L. Nava ${ }^{3}$, V. Neustroev ${ }^{36}$, C. Nigro $^{5}$, K. Nilsson ${ }^{25}$, K. Nishijima ${ }^{32}$, K. Noda $^{6}$, S. Nozaki ${ }^{31}$, Y. Ohtani ${ }^{6}$, T. Oka ${ }^{31}$, J. Otero-Santos ${ }^{1}$, S. Paiano $^{3}$, M. Palatiello ${ }^{2}$, D. Paneque ${ }^{15}$, R. Paoletti ${ }^{13}$, J. M. Paredes ${ }^{18}$, L. Pavletić ${ }^{23}$, P. Peñil ${ }^{9}$, M. Persic ${ }^{2,50}$, M. Pihet ${ }^{15}$, P. G. Prada Moroni ${ }^{17}$, E. Prandini ${ }^{11}$, C. Priyadarshi ${ }^{5}$, I. Puljak ${ }^{29}$, W. Rhode ${ }^{7}$, M. Ribó ${ }^{18}$, J. Rico ${ }^{5}$, C. Righi ${ }^{3}$, A. Rugliancich ${ }^{17}$, N. Sahakyan ${ }^{28}$, T. Saito ${ }^{6}$, S. Sakurai ${ }^{6}$, K. Satalecka ${ }^{14}$, F. G. Saturni ${ }^{3}$, B. Schleicher ${ }^{24}$, K. Schmidt ${ }^{7}$, T. Schweizer ${ }^{15}$, J. Sitarek ${ }^{12}$, I. Šnidaric ${ }^{37}$, D. Sobczynska ${ }^{12}$, A. Spolon ${ }^{11}$, A. Stamerra ${ }^{3}$, J. Striškovic ${ }^{30}$, D. Strom ${ }^{15}$, M. Strzys ${ }^{6}$, Y. Suda ${ }^{27}$, T. Suric ${ }^{37}$, M. Takahashi ${ }^{6}$, R. Takeishi ${ }^{6}$, F. Tavecchio ${ }^{3}$, P. Temnikov ${ }^{34}$, T. Terzić ${ }^{23}$, M. Teshima ${ }^{15,6}$, L. Tosti ${ }^{38}$, S. Truzzi $^{13}$, A. Tutone ${ }^{3}$, S. Ubach ${ }^{26}$, J. van Scherpenberg ${ }^{15}$, G. Vanzo $^{1}$, M. Vazquez Acosta ${ }^{1}$, S. Ventura ${ }^{13}$, V. Verguilov ${ }^{34}$, C. F. Vigorito ${ }^{21}$, V. Vitale ${ }^{39}$, I. Vovk ${ }^{6}$, M. Will ${ }^{15}$, C. Wunderlich ${ }^{13}$, T. Yamamoto $^{40}$, and D. Zarić 29

${ }^{1}$ Instituto de Astrofísica de Canarias and Dpto. de Astrofísica, Universidad de La Laguna, E-38200, La Laguna, Tenerife, Spain ${ }^{2}$ Università di Udine and INFN Trieste, I-33100 Udine, Italy ${ }^{3}$ National Institute for Astrophysics (INAF), I-00136 Rome, Italy ${ }^{4}$ ETH Zürich, CH-8093 Zürich, Switzerland ${ }^{5}$ Institut de Física d'Altes Energies (IFAE), The Barcelona Institute of Science and Technology (BIST), E-08193 Bellaterra (Barcelona), Spain ${ }^{6}$ Japanese MAGIC Group: Institute for Cosmic Ray Research (ICRR), The University of Tokyo, Kashiwa, 277-8582 Chiba, Japan ${ }^{7}$ Technische Universität Dortmund, D-44221 Dortmund, Germany ${ }^{8}$ Croatian MAGIC Group: University of Zagreb, Faculty of Electrical Engineering and Computing (FER), 10000 Zagreb, Croatia ${ }^{9}$ IPARCOS Institute and EMFTEL Department, Universidad Complutense de Madrid, E-28040 Madrid, Spain ${ }^{10}$ Centro Brasileiro de Pesquisas Físicas (CBPF), 22290-180 URCA, Rio de Janeiro (RJ), Brazil ${ }^{11}$ Università di Padova and INFN, I-35131 Padova, Italy ${ }^{12}$ University of Lodz, Faculty of Physics and Applied Informatics, Department of Astrophysics, 90-236 Lodz, Poland ${ }^{13}$ Università di Siena and INFN Pisa, I-53100 Siena, Italy ${ }^{14}$ Deutsches Elektronen-Synchrotron (DESY), D-15738 Zeuthen, Germany ${ }^{15}$ Max-Planck-Institut für Physik, D-80805 München, Germany ${ }^{16}$ Instituto de Astrofísica de Andalucía-CSIC, Glorieta de la Astronomía s/n, 18008, Granada, Spain ${ }^{17}$ Università di Pisa and INFN Pisa, I-56126 Pisa, Italy ${ }^{18}$ Universitat de Barcelona, ICCUB, IEEC-UB, E-08028 Barcelona, Spain ${ }^{19}$ Armenian MAGIC Group: A. Alikhanyan National Science Laboratory, 0036 Yerevan, Armenia ${ }^{20}$ Centro de Investigaciones Energéticas, Medioambientales y Tecnológicas, E-28040 Madrid, Spain ${ }^{21}$ INFN MAGIC Group: INFN Sezione di Torino and Università degli Studi di Torino, I-10125 Torino, Italy ${ }^{22}$ INFN MAGIC Group: INFN Sezione di Bari and Dipartimento Interateneo di Fisica dell'Università e del Politecnico di Bari, I-70125 Bari, Italy ${ }^{23}$ Croatian MAGIC Group: University of Rijeka, Department of Physics, 51000 Rijeka, Croatia ${ }^{24}$ Universität Würzburg, D-97074 Würzburg, Germany ${ }^{25}$ Finnish MAGIC Group: Finnish Centre for Astronomy with ESO, University of Turku, FI-20014 Turku, Finland ${ }^{26}$ Departament de Física, and CERES-IEEC, Universitat Autònoma de Barcelona, E-08193 Bellaterra, Spain ${ }^{27}$ Japanese MAGIC Group: Physics Program, Graduate School of Advanced Science and Engineering, Hiroshima University, 739-8526 Hiroshima, Japan ${ }^{28}$ Armenian MAGIC Group: ICRANet-Armenia at NAS RA, 0019 Yerevan, Armenia ${ }^{29}$ Croatian MAGIC Group: University of Split, Faculty of Electrical Engineering, Mechanical Engineering and Naval Architecture (FESB), 21000 Split, Croatia ${ }^{30}$ Croatian MAGIC Group: Josip Juraj Strossmayer University of Osijek, Department of Physics, 31000 Osijek, Croatia ${ }^{31}$ Japanese MAGIC Group: Department of Physics, Kyoto University, 606-8502 Kyoto, Japan ${ }^{32}$ Japanese MAGIC Group: Department of Physics, Tokai University, Hiratsuka, 259-1292 Kanagawa, Japan ${ }^{33}$ Saha Institute of Nuclear Physics, HBNI, 1/AF Bidhannagar, Salt Lake, Sector-1, Kolkata 700064, India ${ }^{34}$ Inst. for Nucl. Research and Nucl. Energy, Bulgarian Academy of Sciences, BG-1784 Sofia, Bulgaria ${ }^{35}$ Japanese MAGIC Group: Department of Physics, Yamagata University, Yamagata 990-8560, Japan ${ }^{36}$ Finnish MAGIC Group: Astronomy Research Unit, University of Oulu, FI-90014 Oulu, Finland ${ }^{37}$ Croatian MAGIC Group: Ruđer Bošković Institute, 10000 Zagreb, Croatia ${ }^{38}$ INFN MAGIC Group: INFN Sezione di Perugia, I-06123 Perugia, Italy ${ }^{39}$ INFN MAGIC Group: INFN Roma Tor Vergata, I-00133 Roma, Italy ${ }^{40}$ Japanese MAGIC Group: Department of Physics, Konan University, Kobe, Hyogo 6588501, Japan ${ }^{41}$ also at International Center for Relativistic Astrophysics (ICRA), Rome, Italy ${ }^{42}$ now at Department for Physics and Technology, University of Bergen, NO-5020, Norway ${ }^{43}$ now at University of Innsbruck ${ }^{44}$ also at Port d'Informació Científica (PIC), E-08193 Bellaterra (Barcelona), Spain ${ }^{45}$ now at Ruhr-Universität Bochum, Fakultät für Physik und Astronomie, Astronomisches Institut (AIRUB), 44801 Bochum, Germany ${ }^{46}$ now at Department of Astronomy, University of California Berkeley, Berkeley CA $94720{ }^{47}$ also at Dipartimento di Fisica, Università di Trieste, I-34127 Trieste, Italy 49 now at Laboratoire d'Annecy de Physique des Particules (LAPP), CNRS-IN2P3, 74941 Annecy Cedex, France ${ }^{50}$ also at INAF Trieste and Dept. of Physics and Astronomy, University of 
Bologna, Bologna, Italy 